\title{
Characterization of Dynamic Hysteresis Behavior of Ferroelectric Actuators
}

\author{
Felix Wolf ${ }^{1,2}$, Alexander Sutor ${ }^{1}$, Stefan J. Rupitsch ${ }^{1}$, Reinhard Lerch ${ }^{1}$ \\ ${ }^{1}$ Chair of Sensor Technology, University of Erlangen-Nuremberg, Germany \\ Paul-Gordan-Str. 3/5, 91052 Erlangen \\ 2 felix.wolf@Ise.eei.uni-erlangen.de
}

\begin{abstract}
We present a model for the simulation of the hysteretic large-signal behavior of ferroelectric actuators. The model is based on the Preisach operator and takes advantage of an analytic weight function. Here, we concentrate on characterizing the transducers' dynamic behavior. First, the influence of creep and rate-dependence on the polarization signal is investigated by means of measurements on lead zirconate titanate samples. In a second step, the model is extended to describe these phenomena. The weight function is modified to a frequency-dependent formulation with a view to considering the model output for rate-dependent input signals. Creep in the polarization signal is covered by an additional creep operator. A comparison of measurements versus simulations illustrates the benefits of the enhanced model and demonstrates the need of considering creep and rate-dependence separately within the model.
\end{abstract}

\section{Introduction}

Ferroelectric sensors and actuators are widespread in research and industrial applications due to their ability to convert mechanical into electrical energy and vice-versa. The functional parts of such applications are often based on piezoceramic materials like lead zirconate titanate (PZT). The multitude of advantages of these materials is accompanied by a strong hysteretic large-signal behavior, directly related to the high driving levels required for actuators. These non-linearities are provoked by the switching nature of the ferroelectric domains, regions with the same electrical dipole orientation of the underlying crystal unit cells. If a critical threshold is passed for a single domain, it does not only orientate along the electric flux lines but also switch by $90^{\circ}$ as well as $180^{\circ}$. The time-dependence of these incidents and further ion diffusion processes are responsible for the characteristics to be strongly creep-influenced and rate-dependent. Both precise measurements and efficient hysteresis modeling are therefore essential to understand and predict this sophisticated behavior. A common approach to phenomenologically describing the polarization hysteresis are models based on the Preisach operator. However, one of the main drawbacks of such classical Preisachtype models is their inability of considering creep in the transfer characteristics of the modeled system as well as time-dependence of the input signal. Since this is in contrast to real ferroelectric behavior, we focus here on the characterization of these dynamic phenomena.

\section{Measurement}

\section{Measurement Principle}

The first objective was to characterize the dynamic hysteresis behavior of the investigated material by means of measurements of the electrical polarization. These measurements have later been used for the identification of the model parameters. We use a modified Sawyer-Tower circuit [1] for this measuring task. Thereby, a precision capacitor is connected in series between the lower electrode of the sample and 


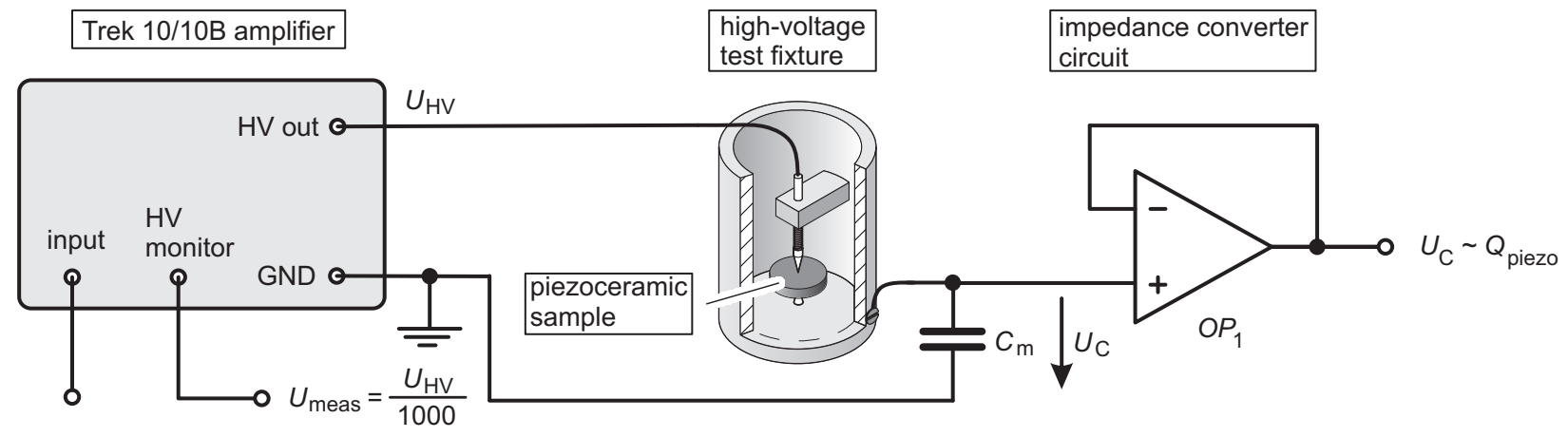

Figure 1: Schematic view of the measurement setup.

ground. This measuring capacitance $C_{\mathrm{m}}$ is chosen much higher than the capacitance of the sample, i.e. $C_{\mathrm{m}} \gg C_{\text {piezo }}(t)$. Therefore, the electrical field strength applied to the specimen can be approximated as

$$
E_{\text {meas }}(t)=\frac{U_{\text {piezo }}(t)}{l} \approx \frac{U_{\text {piezo }}(t)+U_{\mathrm{C}}(t)}{l}=\frac{U_{\mathrm{HV}}(t)}{l}=\frac{U_{\text {meas }}(t) \cdot 1000}{l},
$$

with $U_{\text {meas }}(t)$ representing the excitation voltage, directly obtained by the voltage monitor output of the Trek 10/10B amplifier (Fig. 1). The variable $l$ denotes the distance of the plane-parallel electrodes of the sample. The challenge is now, how to measure the voltage $U_{\mathrm{C}}(t)$ across the capacitance $C_{\mathrm{m}}$. A very high

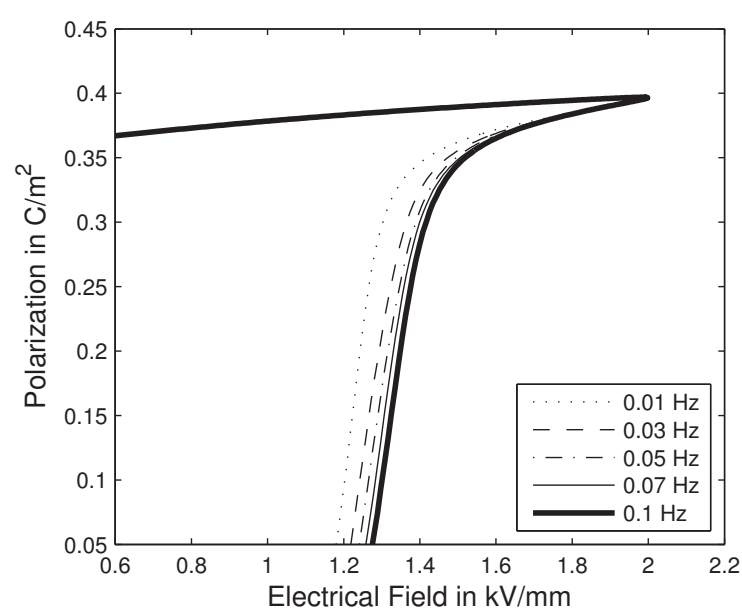

(a)

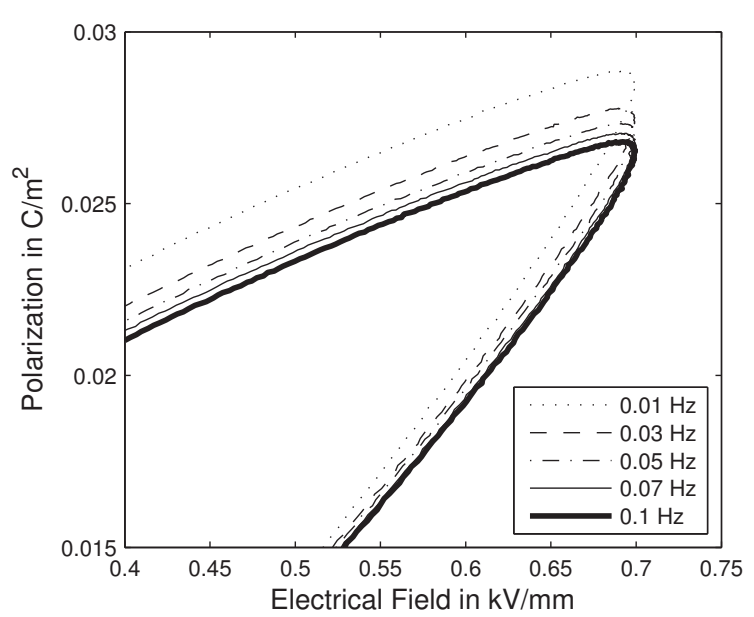

(b)

Figure 2: Measured major loops (a) and minor loops (b) for sinusoidal excitation of different frequencies.

input resistance of the measuring unit is required for this task to prevent the capacitor from discharging and thus distorting the measured signal. We use an impedance converter circuit with an operational amplifier TL081 $\left(O P_{1}\right)$ offering a theoretical input resistance of $10^{12} \Omega$. The electric charge $Q_{\mathrm{C}}(t)$ on the precision capacitor can therefore be considered as equal to the charge $Q_{\text {piezo }}(t)$ on the electrodes (with surface area $A$ ) of the sample. Thus the polarization is calculated as

$$
P_{\text {meas }}(t)=\frac{Q_{\text {piezo }}(t)}{A}-\varepsilon_{0} \cdot E_{\text {meas }}(t) \approx \frac{U_{\mathrm{C}}(t) \cdot C_{\mathrm{m}}}{A}-\varepsilon_{0} \cdot E_{\text {meas }}(t)
$$

where $\varepsilon_{0}$ depicts the vacuum permittivity. The sample is placed in an oil bath to prevent from dielectric breakdown. It is fixed by a spring tip electrode to allow free oscillation (Fig.1). The excitation signal is numerically generated and amplified by the high-voltage amplifier Trek 10/10B. Both measured signals, $U_{\text {meas }}(t)$ and $U_{\text {piezo }}(t)$ are converted to digital signals and evaluated on a measuring computer. 


\section{Measurement Results}

The measurements presented in this paper have been performed for discoidal samples out of Pz27, a soft lead zirconate titanate material manufactured by Ferroperm Piezoceramics A/S. The samples have a thickness $l$ of $2 \mathrm{~mm}$, a diameter of $25 \mathrm{~mm}$ and feature plane-parallel silver electrodes on their frontfaces. In order to investigate the rate-dependence, we applied sinusoidal excitation signals of ten uniformly

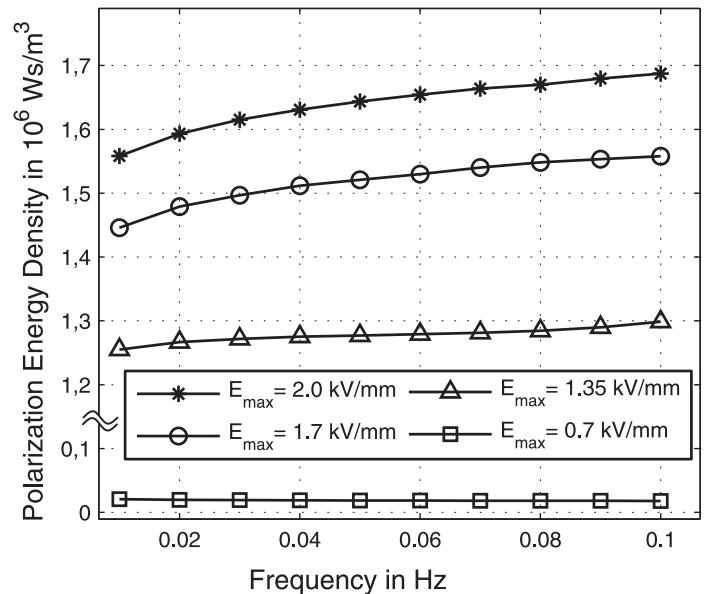

(a)

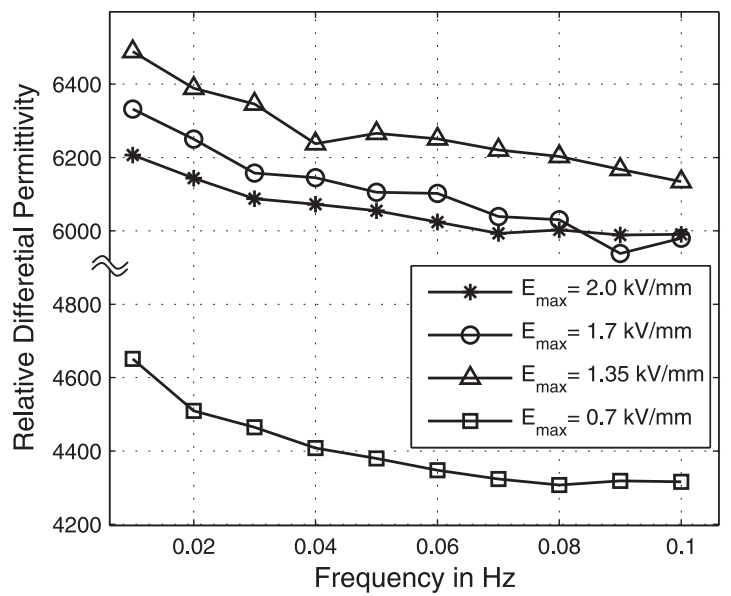

(b)

Figure 3: Frequency-dependent polarization energy density (a) and relative differential permittivity (b) for different maximum field strengths.

distributed frequencies between 0.01 and $0.1 \mathrm{~Hz}$ and at four different maximum field strengths $(0.7,1.35$, 1.7 and $2.0 \mathrm{kV} / \mathrm{mm})$. Whereas the major loops $(2.0 \mathrm{kV} / \mathrm{mm}$, Fig. $2(\mathrm{a}))$ show significant saturation behavior, this effect is reduced for loops with lower maximum field strengths. For minor loops $(0.7 \mathrm{kV} / \mathrm{mm}$, Fig. 2(b)), no saturation can be observed at all. As clearly can be seen, the frequency of the input signal is mainly affecting the coercive field strength of major loops. The dominant influence on minor loops is instead the polarization amplitude, decreasing with frequency. Figure 3(a) shows the polarization energy density, also denoted as dissipation energy density. This value is directly related to the self-heating of the sample. The polarization energy density, calculated as the area inside the hysteresis loop, increases with higher frequencies for major loops. For minor loops, it is even slightly decreasing with frequency. This indicates that both, maximum amplitude and the excitation frequency determine the occurrence of domain switching incidents. The relative differential permittivity $\varepsilon_{d, r}^{T}=\frac{\Delta P}{\Delta E} \cdot \varepsilon_{0}{ }^{-1}$ depicts the slope of the hysteresis close to remanent polarization, divided by the vacuum permittivity. Its frequency-dependent variation is illustrated in Fig.3(b). The relative differential permittivity decreases for all amplitudes of the electrical field with frequency. This implies that the excitation frequency has a significant influence on the dielectric properties of the investigated material.

\section{Modeling}

In our previous work $[2,3,4,5]$, we proposed a phenomenological model for the ferroelectric polarization hysteresis taking into account the Preisach hysteresis operator $\mathcal{H}[6]$

$$
P_{\text {model }, \mathrm{n}}(t)=\mathcal{H}\left[E_{\mathrm{n}}\right](t)=\iint_{\alpha \geq \beta} \mu(\alpha, \beta) \gamma_{\alpha \beta}\left[E_{\mathrm{n}}\right](t) \mathrm{d} \alpha \mathrm{d} \beta .
$$

The electrical polarization $P_{\text {model, } \mathrm{n}}(t)$ for a given electrical field $E_{\mathrm{n}}(t)$ is calculated by superimposing a set of fundamental switching operators $\gamma_{\alpha \beta}$. These switching operators can only feature two distinct values (-1 and 1), depending on the thresholds $\alpha$ and $\beta$. Input and output of the Preisach operator are both normalized to the maximum value. A significant part of the model is the weight function $\mu(\alpha, \beta)$, mainly affecting the 
shape of the hysteresis loops. Contrary to common approaches [7, 8], we use an analytic weight function $\mu_{D A T}(\alpha, \beta)$ within the model

$$
\mu_{D A T}(\alpha, \beta)=\frac{B}{1+\left\{\left[(\alpha+\beta) \sigma_{1}\right]^{2}+\left[(\alpha-\beta-h) \sigma_{2}\right]^{2}\right\}^{\eta}} .
$$

Apart from reducing the computational effort, another benefit of such an analytic weight function is the ability of considering environmental conditions like temperature, pre-stress and dynamic properties. The model parameters are identified by matching the model output to measurements. A more detailed discussion of Preisach models in general and about our model can be found in literature [2, 3, 4, 5, 9].

In terms of dynamic hysteresis modeling, creep and rate-dependence have to be treated separately to our opinion. Both result in a different impact on the shape of the polarization hysteresis loops. We therefore consider creep by an additional creep operator whereas the rate-dependence is covered by a frequencydependent formulation $\mu_{D A T}(\alpha, \beta, f)$ of the weight function.

\section{Creep Operator}

As discussed in detail in our previous work [5], the investigated piezoceramic material shows an aperiodic step-response when applying an appropriate step signal of the electrical field. Motivated by this low-pass characteristics, the creep in the polarization signal is described as the solution of the first-order differential equation

$$
\frac{\mathrm{d}}{\mathrm{d} t} P_{\text {creep }, \mathrm{n}}(t)-\alpha_{D} \cdot\left(P_{\mathrm{n}}(t)-P_{\text {creep }, \mathrm{n}}(t)\right)=0 .
$$

The creep-affected polarization signal is thus obtained by applying an additional creep operator $\mathcal{D}$ to the

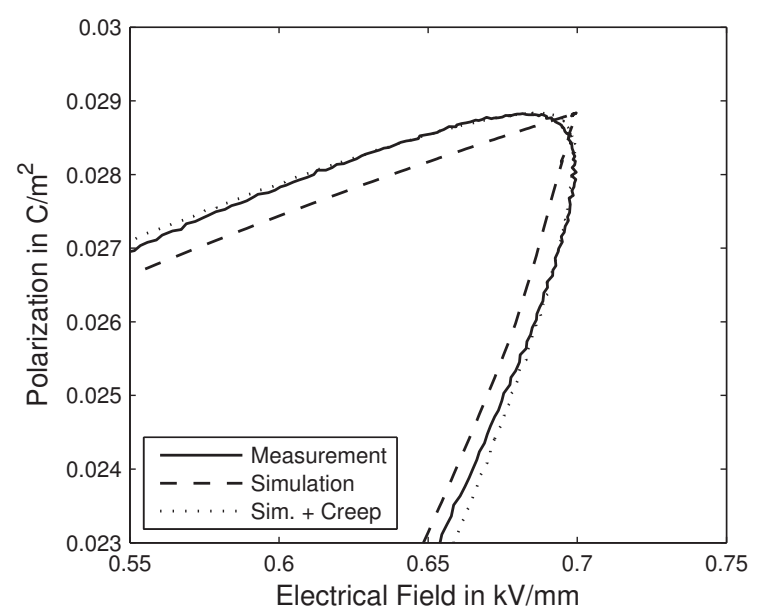

(a)

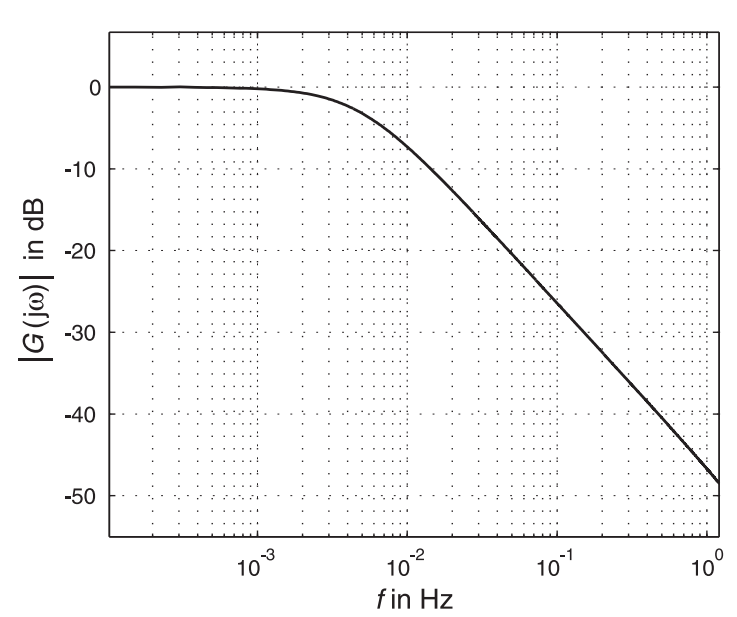

(b)

Figure 4: Minor loop compared to simulation with and without creep operator for $\alpha_{D}=0.03 \mathrm{~s}^{-1}$ (a). Absolute value of transfer function $G(\mathrm{j} \omega)$ of creep operator for $\alpha_{D}=0.03 \mathrm{~s}^{-1}(\mathrm{~b})$.

output of the Preisach operator

$$
P_{\text {creep }, \mathrm{n}}(t)=\mathcal{D}\left[P_{\text {model }, \mathrm{n}}\right](t)=\mathcal{D}\left[\mathcal{H}\left[E_{\mathrm{n}}\right]\right](t)=P_{0} e^{-\alpha_{D}\left(t-t_{0}\right)}+\int_{t_{0}}^{t} \alpha_{D} e^{-\alpha_{D}(t-\tau)} P_{\text {model }, \mathrm{n}}(\tau) \mathrm{d} \tau,
$$

with the initial value $P_{0}$ and the creep parameter $\alpha_{D}$. The enhanced model is compared to the model without creep operator in Fig. 4(a). The smooth turning points of the minor loops can now be simulated very well. But obviously, it is not possible to describe the rate-dependent loops as observed in section 2 with just a single parameter $\alpha_{D}$. An additional description of the frequency dependence is indispensable. The absolute value of the transfer function

$$
G(\mathrm{j} \omega)=\frac{P_{\text {creep }}(\mathrm{j} \omega)}{P(\mathrm{j} \omega)},
$$


determined for the same $\alpha_{D}=0.03 \mathrm{~s}^{-1}$ as used for the simulation of the creep-dependent minor loop in Fig. 4(a) is illustrated in Fig. 4(b). It reveals that only very low frequencies have to be considered when describing the frequency-dependence of hysteresis loops. For higher frequencies, the damping nature of the creep operator is the dominant phenomenon.

\section{Frequency-dependent Weight Function}

The impact of the excitation frequency on measured hysteresis loops has already been described in section 2. For major loops, the coercive field strength is mainly affected, whereas the amplitude is also altered

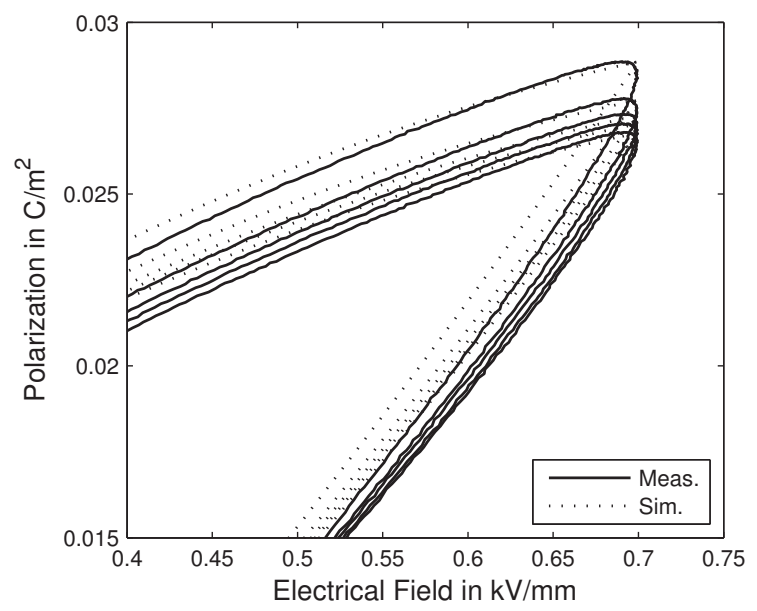

(a)

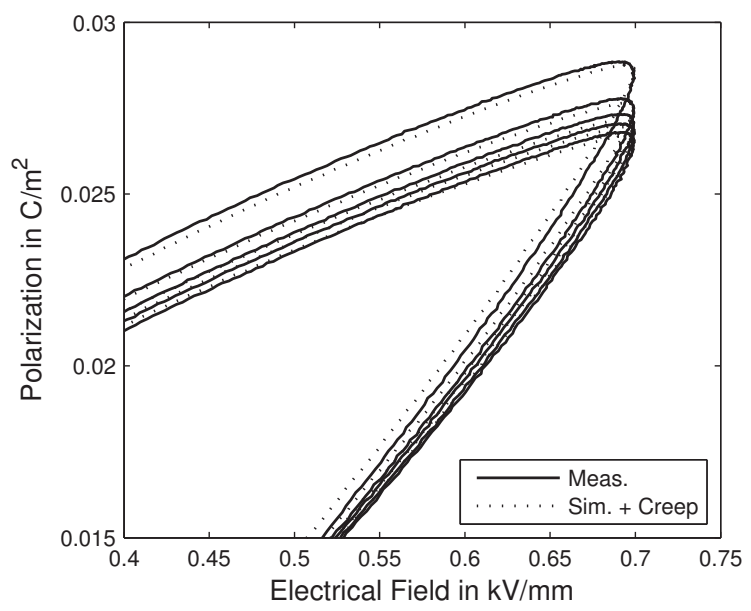

(b)

Figure 5: Measurements of minor loops at different frequencies compared to simulations with frequencydependent formulation of weight function. The simulations have been performed with (b) and without (a) Creep-Operator.

for minor loops. A similar, but linear influence can be observed for simulations with a variation of the parameter $h$. This leads to a frequency-dependent formulation of the analytic weight function [10]

$$
\mu_{D A T}(\alpha, \beta, f)=\frac{B}{1+\left\{\left[(\alpha+\beta) \sigma_{1}\right]^{2}+\left[\left(\alpha-\beta-\left(\nu_{1}-\frac{\nu_{2}}{f^{\nu_{3}}}\right)\right) \sigma_{2}\right]^{2}\right\}^{\eta}},
$$

where $h$ was replaced by the additional rate-dependent function

$$
h(f)=\nu_{1}-\frac{\nu_{2}}{f \nu_{3}} .
$$

This analytic function directly describes the variation of the measured coercive field strength of major loops with frequency. In addition, the same function is also mapping the frequency-induced decrease of the maximum polarization of minor loops (Fig. 6(a)). Simulations, performed with the rate-dependent model are compared to measurements in Fig. 5(a) and Fig.6(b). For major loops, the model output fits quite well to the measurements, apart from the small range, where the polarization reaches saturation. For minor loops, the resulting sharp edges of the simulated signal lead to significant deviations in the polarization signal (Fig. 5(a)). The model output for the combination of creep operator and rate-dependent weight function is displayed in Fig. 5(b). The simulation results for minor loops are improved substantially, if the creep operator is applied additionally. However, the influence of the creep operator on major loops can be neglected. With three additional parameters, the dynamic behavior, including creep and rate-dependence is thus described very well.

\section{Conclusion}

A model for the simulation of the dynamic polarization hysteresis of ferroelectric actuators was presented. Simulations, compared to measurements reveal that both, the creep operator and the frequency depen- 

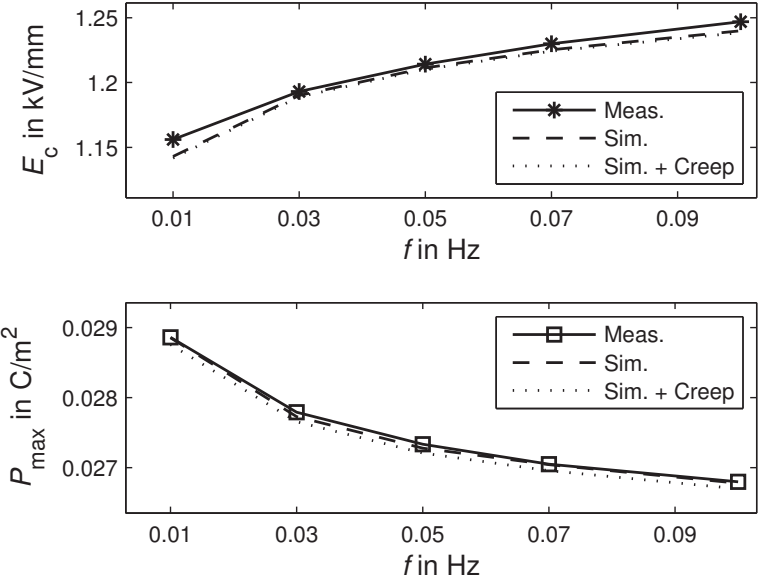

(a)

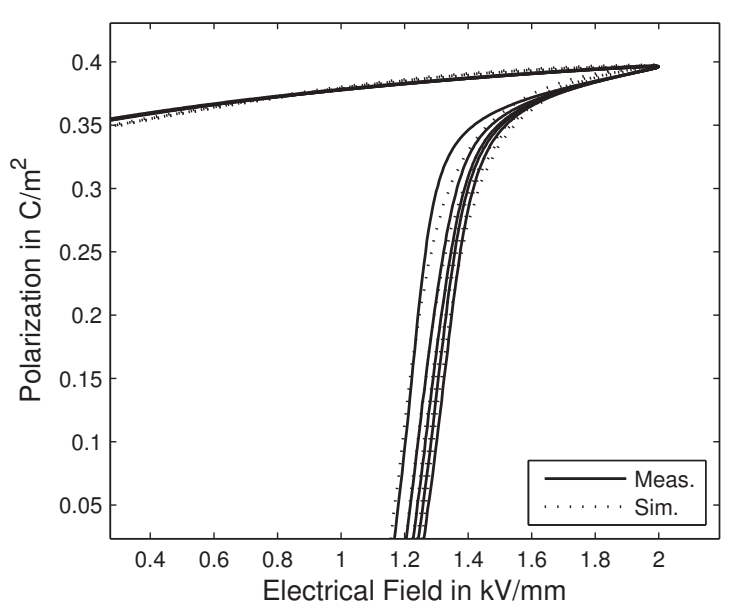

(b)

Figure 6: Frequency-dependent coercive field strength $E_{\mathrm{c}}(f)$ for major loops and maximum polarization $P_{\max }(f)$ for minor loops - measurement compared to simulations (a). Measurements of major loops at different frequencies compared to simulations (b).

dent formulation of the weight function are essential to describe the whole dynamic behavior. Whereas there are still little deviations for major loops close to saturation, the model allows now excellent simulations of frequency-dependent and creep-influenced minor loops. Thus the dynamic polarization in the working range of actuators can be simulated with only three additional parameters. Future research is concentrated on investigating multi-frequent signals by considering the dominant frequencies for the rate-dependent formulation.

\section{Acknowledgement}

The underlying research is gratefully supported by the German Research Foundation (DFG) as part of the special research field SFB/TR39.

\section{References}

[1] C. B. Sawyer and C. H. Tower. Rochelle salt as a dielectric. Physical Review, 35(3):269-273, 1930.

[2] A. Sutor and R. Lerch. A Preisach Based Model for the Characterisation of Magnetic Hysteresis. In Sensors 2009, Nürnberg, 2009.

[3] A. Sutor, S. J. Rupitsch, and R. Lerch. A preisach-based hysteresis model for magnetic and ferroelectric hysteresis. Applied Physics A: Materials Science and Processing, 100(2):425-430, 2010.

[4] F. Wolf, A. Sutor, S. J. Rupitsch, and R. Lerch. Messtechnische Erfassung und Modellierung der Hysterese Ferroelektrischer Werkstoffe. In Sensoren und Messsysteme 2010, pages 421-424, Nürnberg, 2010.

[5] F. Wolf, A. Sutor, S. J. Rupitsch, and R. Lerch. Modeling and measurement of hysteresis of ferroelectric actuators considering time-dependent behavior. Procedia Engineering, 5:87-90, 2010.

[6] F. Preisach. Über die magnetische Nachwirkung. Zeitschrift für Physik, 94(5-6):277-302, 1935.

[7] T. Hegewald, B. Kaltenbacher, M. Kaltenbacher, and R. Lerch. Efficient modeling of ferroelectric behavior for the analysis of piezoceramic actuators. Journal of Intelligent Material Systems and Structures, 19:1117-1129, 2008.

[8] M. Kaltenbacher, B. Kaltenbacher, T. Hegewald, and R. Lerch. Finite element formulation for ferroelectric hysteresis of piezoelectric materials. Journal of Intelligent Material Systems and Structures, 21:773-785, 2010.

[9] I.D. Mayergoyz. Mathematical Models of Hysteresis and Their Applications. Elsevier, New York, 2003.

[10] F. Wolf, A. Sutor, S. J. Rupitsch, and R. Lerch. Modeling and measurement of creep- and rate-dependent hysteresis in ferroelectric actuators. Sensors and Actuators A, 2011. Article in Press. 